\title{
Melancolía, quebranto, tensión y disfrute Representaciones sociales respecto de las experiencias de construcción de la vivienda en los barrios populares periféricos
}

\section{MARÍA MANEIRO}

mariamaneiropinhero@gmail.com - Instituto de Investigaciones Gino Germani, Universidad de Buenos Aires (UBA) / Consejo Nacional de Investigaciones Científicas y Técnicas (CONICET), Argentina

Fecha de recepción: 20 de abril de 2017

Fecha de aceptación: 31 de mayo de 2017

\section{RESUMEN}

La vivienda se ha evidenciado como un problema social de envergadura. Uno de los asuntos a considerar acerca del porqué de este problema es que éste no se constituyó como agente relevante de construcción de planes de vivienda. Es así como la responsabilidad en torno a la construcción de la morada ha signado ala población. La producción dela vivienda constituye un desafío costoso. Partimos del supuesto de quelainvestiduraqueésta contieneresultacentral para entender el dicho proceso. En este artículo se abordan las representaciones sociales enunciadas en cuatro memorias biográficas de los habitantes de un barrio popular acerca del acceso, el proceso de producción y el estatuto de la vivienda propia. Para ello se indagaron las remisiones en torno al valor de uso y al valor simbólico prestando atención a la temporalidad. La base empírica de esta exploración está centrada en una serie de entrevistas abiertas realizadas durante el año 2015 en un barrio popular periférico del partido de Quilmes, en el Área Metropolitana de Buenos Aires, Argentina.

PALABRASCLAVES: barriopopularperiférico, vivienda, autoconstrucción, catexis, representaciones sociales.

\section{ABSTRACT}

Housing has shown to be a major social problem. One of the issues to consider regarding the cause of this problem is that the state was not constituted as a relevant agent for the construction of housing plans. Therefore, the responsibility surrounding the construction of homes has marked the population. The production of housing constitutes an expensive challenge. We assume that the cathexis of housing is key to understand such process. This article addresses the social representations stated in four biographic memoirs of the dwellers of a working-class neighborhood about the access, the production process and the statute of home ownership. For such purpose, the references to the use value and the symbolic value have been investigated paying attention to temporariness. This exploration's empiric foundation focuses on a series of open interviews made during year 2015 in a peripheral working-class neighborhood of the Quilmes district, in the Buenos Aires Metropolitan Area, Argentina.

KEY WORDS: peripheral working class neighborhood, housing, self-construction, cathexis, social representations. 


\section{INTRODUCCIÓN}

Dentro del Área Metropolitana de Buenos Aires (AMBA) se han desarrollado formas diferenciales de acceso a la vivienda y de configuración espacial delos entramados territoriales (Di Virgilio, 2015; Varela \& Cravino, 2009). Los sectores populares - aunque no solo ellos- han accedido mayoritariamente a la tierra mediante mecanismos informales (Herzer, Di Virgilio, Rodríguez \& Redondo, 2008) y han sido los promotores y hacedores directos de la construcción de sus viviendas.

En este artículo partimos de un supuesto: se ha instituido una investidura afectiva de gran magnitud en lo que hace a la producción de la vivienda propia en quienes habitan los barrios periféricos del conurbano. Dicha investidura fue el motor del esfuerzo vital, económico y relacional que llevaron adelante los habitantes de los barrios donde investigamos. Si bien existen itinerarios exitosos y quebrantados respecto de este desafío, entendemos que éstos no invalidan el supuesto mencionado. Bajo este paraguas, aquíse pretende rastrear las representaciones sociales que emergen en los pobladores, respecto de sus propias viviendas, a partir de explorar las remisiones a la cuestión del valor.

Con este norte, en primer término, se intentará echar luz acerca de cómo aparece en la memoria de nuestros entrevistados el acceso al barrio, al terreno $\mathrm{y}$, si fuera el caso, a la vivienda. Exploraremos el valor de este evento ylas formas en que se lleva a cabo.

En segundo término, indagaremos las representaciones en torno a la primera figuración con la que se produce la vivienda: la casilla; entroncada a la memoria de la urgencia y de la necesidad, esta figura es la que prontamente sutura el valor de uso de la vivienda. No obstante, en las representaciones de los entrevistados, la casilla no constituye una huella olvidada de aquello que fue, sino que posee una red de significados actuales que intentaremos describir en las páginas que siguen, articulando en sí un fuerte valor simbólico.

En tercer término, trabajaremos las representaciones en torno al valor de uso y el valor simbólico de la vivienda propiamente dicha (la casa de material); no obstante éste no es un valor ya dado, sino un valor a producir: la trasformación del terreno, la construcción trabajosa de la vivienda y el ascetismo de la vida en construcción conforman un magma inacabado de significaciones. Es en este sentido que dicho valor se encuentra ligado - en forma ambigua y compleja - con el placer y el hastío (la casa como espacio/proyecto de placer, la producción de la casa como proceso agobiante); dentro de este mismo aspecto, cabe atender a la multiplicación de partícipes que engloba la vivienda: las representaciones la subdivisión parcelaria yla cohabitación adquieren aquí un lugar de relevancia. Todos estos aspectos se encuentran cortados transversalmente por la historicidad, la larga duración de cada una de estas hechuras y permanencias construyen y corroen valoraciones en torno al bien que nos ocupa. 


\section{ALGUNAS HERRAMIENTAS TEÓRICAS}

En este apartado se precisan sucintamente los nudos analíticos que guían este artículo. En primer lugar, explicitaremos los ejes que subyacen en nuestros supuestos. A pesar de que la construcción de la vivienda propia supone un gasto energético yeconómico de envergadura ${ }^{1}$, antela escasez de políticas públicas, esta modalidad se ha generalizado entre los sectores populares. Entendemos que ésta se encuentra movilizada por una carga afectiva (investidura o catexis, en términos psicoanalíticos) que subsume la necesidad del bien material vivienda. Ésta produce un plus de vitalidad en sus propios hacedores. Sin embargo, existen circunstancias sociales que quiebran esta energía. En este artículo proponemos cuatro experiencias diferentes que permiten entender la complejidad de este asunto.

Algunos enfoques anclados en lo que se ha dado en llamar el giro afectivo han situado sus abordajes desde una perspectiva que se distancia de los estudios enmarcados en el lenguaje y la representación social ${ }^{2}$. Si bien tomamos nota del recorte y los límites que estas mediaciones instituyen, nuestro prisma posee como eje central el lenguaje. Entendemos, siguiendo a Scott (2001), que la experiencia no supone un dato exterior a la discursividad, sino que es reelaborada por los sujetos desde el presente, construyendo memorias biográficas que emergen como representaciones sociales. La noción de representación social retoma los desarrollos de Denise Jodelet (1986). Para ella una representación social es una forma de presentación discursiva de la realidad social, que implica una construcción activa y creativa de un sujeto socializado; ésta no es el puro reflejo interior de algo exterior, sino una modalidad específica de presentar mediante el lenguaje la realidad social acorde a las experiencias de socialización que cada sujeto haya vivido. Toda representación social se compone de información y actitud(ovalor), configurandoun núcleo figurativo. Muchos delosaspectosquese trabajan en esta presentación suponen una reelaboración singular de procesos pasados, en este sentido se entiende que remiten al campo de las memorias ${ }^{3}$.

Resulta pertinente clarificar los vectores analíticos en los que se asienta la noción de valor. Un punto de partida para pensar esta noción se inscribe en la propuesta marxista en torno a la diferenciación entre el valor de cambio y el valor de uso (como aspectos constitutivos de la mercancía) 4 . Aquí se ha retomado parcialmente esta forma de concebir los valores puesto que sela ha interceptado con la cuestión de la afectividad, del valor simbólico y de la temporalidad.

1 Una aproximación sugerente en torno a esta cuestión se puede ver en el texto de García García (2005).

2 Tal como he mencionado arriba, nuestro trabajo, a pesar de trabajar con aspectos afectivos, no se enmarca en el llamado giro afectivo. En torno a esta perspectiva y a su distancia con los estudios eslabonados a partir de las representaciones sociales y el lenguaje se puede visitar en el artículo de Ali, Lara y Giazu Enciso Domínguez (2013).

3 Siguiendo los aportes de Bertotti (2015), entendemos a las memorias como un subgrupo de las representaciones sociales, cuyo objeto de representación remite a procesos y sucesos pretéritos. Sin embargo, la significación se encuentra ligada a las modalidades de representación actual.

4 Ciertamente, esta diferenciación se asienta en el abordaje marxista desarrollado en el primer capítulo de El Capital, Marx, ([1867] 2010). No obstante, una revisión y actualización de este enfoque inscripto en el problema que nos ocupa se puede encontrar en "Contradicción 1. Valor de uso y valor de cambio" (Harvey, 2014). 
Desde la perspectiva clásica, la remisión al valor de uso de una mercancía se encuentra asociado a la utilidad y a la necesidad, en este caso, de la vivienda propia; sin embargo es menester aclarar que la producción misma de la vivienda como bien de uso constituye un complejo y demorado proceso que puede modificar su valor a lo largo de su biografía (Appadurai, 1991); éste se compone de vastas capas que se estiman y se significan de modos diversos. Dentro dela noción de valor de uso existe una remisión particular, que adquiere cierta especificidad y que podría entenderse retomando el concepto de valor simbólico ${ }^{5}$; tomamos el sentido de este concepto de García Canclini (1993, p. 34), entendiendo que en ciertos casos el proceso de apropiación y uso de determinados objetos expresa una cierta prevalencia sobre los valores de uso y de cambio, o donde al menos estos últimos se configuran subordinados a la dimensión simbólica ${ }^{6}$. En este sentido, la carga afectiva y la cualidad del apego son los que caracterizan este tipo de valor.

Por otra parte, la dimensión referida al valor de cambio, que en la propuesta marxista refiere a la abstracción que posibilita el intercambio, no será explorada en este artículo puesto que el análisis delas entrevistas no mostró una apelación afectivamente connotada respecto de este aspecto.

\section{PRECISIONES METODOLÓGICAS}

Esta presentación tiene como sustento empírico una serie de 13 entrevistas abiertas realizadas en el municipio de Quilmes. El partido de Quilmes, se encuentra inserto en el segundo cordón del conurbano bonaerense, a $21 \mathrm{~km}$. de la Ciudad Autónoma de Buenos Aires. Su composición socio habitacional presenta un rasgo distintivo: la informalidad. Para mediados de la primera década de este siglo, el $47 \%$ de la población del distrito se encontraba marcada por algún tipo de informalidad (Núñez, 2004). Según datos del Censo Social realizado por el municipio, para el año 2010, 101.028 personas viven en zonas de villas o asentamientos precarios, lo cual suponequeel $18,4 \%$ dela población de Quilmes se encuentra en situación de vulnerabilidad residencial (Censo Social, 2010).

El barrio Alicia Esther y el barrio El Tala, donde fueron realizadas las entrevistas que se analizan en este trabajo, se ubican dentro de estas zonas de vulnerabilidad. El primero de estos dos barrios es resultado de un loteo producido durante los primeros años de la década del '70; no obstante las últimas adquisiciones de terrenos se efectuaron con posterioridad a la inhibición de este tipo de operaciones, produciéndose la compra por fuera de los marcos de la legislación vigente. El barrio El Tala, inscripto en la serie de asentamientos

5 Harvey incluye al valor simbólico como uno de los elementos posibles del valor de uso. Nosotros incluimos dicho aspecto dentro de este valor, pero le otorgamos una entidad particular.

6 Conocemos las discusiones que esta definición contrae en la medida en que se dificulta medir el quantum de prevalencia entre un valor y otro, sin embargo consideramos que resulta útil para echar luz en los aspectos que estamos intentando dilucidar. 
surgidos a partir de la ocupación de tierras de comienzos de la década del ' 80 , se engendra a partir de una organización comunitaria con la colaboración de la Iglesia Católica. Ambos entramados residenciales se encuentran localizados en la frontera entre los municipios de Quilmes y Almirante Brown; y a pesar de sus génesis diferenciales, la imagen actual no presenta diferencias urbanísticas entre ambos. Los dos contienen terrenos con viviendas autoconstruidas que mayoritariamente contienen más de un enclave habitacional, no existen espacios verdes y el principal problema de la zona son las frecuentes inundaciones.

Las entrevistas se realizaron durante el mes de noviembre de $2015^{7}$. Dicho trabajo de campo dio como resultado un conjunto de entrevistas conformadas a partir de una muestra en la que se controlaba el sexo y la edad. A partir de una lectura exhaustiva de las mismas, para este artículo en particular se seleccionaron cuatro entrevistas de la generación adulta (que poseía de 31 a 60 años en el momento del trabajo de campo). El enfoque de esta generación posibilita profundizar la dimensión experiencial —en relación a la adquisición de la vivienda - en la configuración representacional de los sujetos entrevistados ${ }^{\mathbf{8}}$. Interrogamos a estos sujetos que desarrollaron su vida adulta en un entorno que habilitaba una diversidad de oportunidades y dificultades en torno a la adquisición y la formalización de la vivienda propia.

La selección de las entrevistas utilizadas para este análisis estuvo guiada por la construcción de una muestra basada en la heterogeneización del corpus, con el objeto de acercarse a los estudios que rastrean las distinciones en las valoraciones sociales dentro de fracciones insertas en los mismos territorios sociales ${ }^{9}$.

Los criterios considerados para la construcción de la muestra son: divergencias respecto de las formas de acceso y modalidad de producción de la vivienda; experiencias laborales propias y familiares, cuestiones generacionales (dentro del recorte etario) y la dimensión de género.

La elección de un grupo pequeño de entrevistas posibilitó el seguimiento de la lógica argumental de cada uno de los cuatro entrevistados y la ampliación de los fragmentos textuales expuestos; asimismo un análisis general delas trece entrevistas realizadas mostró que las formas representacionales de los otros entrevistados pueden entenderse a partir de los núcleos figurativos que resultaron de los casos analizados para este artículo.

7 Ficha técnica del trabajo de campo. Fecha:7/11/2015. Barrios El Tala/Alicia Esther Municipio: Quilmes. Seminario: Procesos de (des)encaje y movimientos sociales: reconfiguraciones de las identidades colectivas. Equipo docente: Maneiro, María; Bertotti, María Carla; Nardin, Santiago. Estudiantes: Brizuela, Carolina; Maidana, Mercedes; Martínez, Paula; Palmucci, Juan Pablo y Rodríguez, Ana María. Apoyo: Wolpowicz, Julián. Entrevistas abiertas. Cantidad de entrevistas realizadas: 13

8 Un primer acercamiento a las entrevistas de los más jóvenes nos mostró que sus itinerarios y representaciones contienen parecidos de familia con los casos de Rosa y Viviana, aunque en ellos se debilita la cuestión de género y se eclipsa la génesis de adquisición de la vivienda por la mediación de una nueva generación. Tal eclipse es lo que nos decidió a desestimarlos para este trabajo. Mayores precisiones en torno a las representaciones sociales de los jóvenes serán abordadas en posteriores artículos.

9 Acerca de esta cuestión, véase: Murard \& Laé (2013). 


\section{ALGUNOS RESULTADOS}

\section{-Rosa: la vivienda, su investidura parental y la melancolía}

Rosa tiene 42 años. No terminó la escuela secundaria. Trabaja como empleada doméstica, no tiene pareja y cría un niño. Ellos habitan una casilla que se ubica en la parte trasera de la casa en la que vive su padre, su hermana y su sobrino. Tanto la vivienda principal como la casilla fueron construidas por sus progenitores y la adquisición del predio se produjo mediante una venta irregular realizada por una inmobiliaria fraguada. Su padre trabajó en la empresa Austral de aeronavegación, con una contratación estable.

Rosa evoca que sus padres compraron este terreno cuando ella tenía dos años. Se lo compraron a una dudosa inmobiliaria que ofrecía lotes en cuotas y ante la menor falta de pago brindaba el terreno a un nuevo comprador.

Pregunta $(\mathrm{P})$ : ¿Cómo llegaron al barrio, dónde estaban viviendo antes? Respuesta (R): Nosotros vivíamos allá en Pacheco y antes en Tucumán. De allá vinimos acá a La Paz y de ahí saltamos acá.

$\mathrm{P}$ : ¿Y por qué vinieron acá, tenían conocidos o familiares?

$\mathrm{R}$ : No, antes vendían terrenos. Acá era un descampado antes. En la esquina (señala hacia Donato ${ }^{10}$ ) se ponían ahí con una mesita y te anotaban (...). Había un... Estudio Blanco, que está en Avellaneda, que vendía los terrenos y... te atrasabas una cuota y te apuraban y muchos salían, y después selos vendían a otros yasí(...) El que se atrasaba, tenían la orden de comunicar allá a la oficina, y si no podías pagar te sacaban (...) todos los meses te aumentaban la cuota, te aumentaban, te aumentaban y la gente... Y sí. Por suerte él trabajaba en Austral y pudimos pagar.

Dos aspectos relevantes surgen de la evocación de Rosa. Ella remite a los loteos populares dela década del ' 70 .Éste, sin embargo, apareceligado a una fuerte discrecionalidad dela inmobiliaria, lo que da cuenta dela informalidad en torno a las reglas que lo establecen ${ }^{11}$. Es llamativo, en relación a las representaciones de los demás vecinos, que Rosa no remita la llegada al barrio a ninguna relación previa. En esta familia es la mediación mercantil la que la hace arribar. Sin embargo, la mediación mercantil que evoca está permeada por la trasgresión ${ }^{12}$.

P: Y ahí [se] descubrió que la gente esa le sacaba plata a la gente, digamos, que cobraba una chequera y nada que ver, ellos no eran dueños de los terrenos.

R: Nadie era el dueño de los terrenos. Nadie era dueño, porque acá supuestamente, era baldío.

En el momento de la escrituración de los terrenos se descubrióla ilegalidad

10 Donato Álvarez es la avenida más importante de la zona y es la que separa jurisdiccionalmente el municipio de Almirante Brown de Quilmes.

11 Una discusión relevante en torno a nuestros datos es la fracasada acción del mercado frente a la exitosa capacidad de las redes de sociabilidad local en las actividades relativas al logro de la vivienda. Este aspecto será trabajado en otra presentación.

12 La lagunaridad estatal articulada a la necesidad y el deseo de tener un terreno pueden ser elementos a considerar para entender la relevancia de este tipo de estafas. 
de la venta de éstos, puesto que los vendedores no acreditaban su propiedad; este aspecto dificulta su regularización. A través de la organización barrial y el preponderante papel de una referente comunitaria, la familia de la entrevistada logra la titularidad de la vivienda. Los lazos barriales se densifican a partir de este proceso, lo cual potencia el apego al bien. Como la mayor parte de los entrevistados, los padres de Rosa llegan a su terreno y plantan su casilla:

R: Nosotros vinimos y plantamos una casilla acá. La pusimos allá atrás y después empezamos a modificar aquí adelante, de a poquito.

La forma instituida del primer habitar en el barrio es construir la casilla ${ }^{\mathbf{1 3}} \mathrm{en}$ la parte trasera del terreno y poco a poco ir construyendo la casa de materiales sólidos y durables adelante. Rosa era una niña cuando sus padres comienzan este proceso, sin embargo en su relato ella es una participante activa del proceso de plantación de la primera vivienda. La casilla es el primer lugar habitado por la familia, el primer hogar donde se condensan las marcas afectivas pretéritas y el recurso familiar frente a nuevas generaciones o contingencias familiares.

R: No, esa casilla era de mi hija, y mi hija me la dio a mí, entonces yo cerré ahí, pero ahora se me está viniendo abajo la casilla y voy a ver si puedo edificar arriba, pero me cuesta mucho.

La casilla donde actualmente vive la entrevistada es la misma que históricamente plantó con sus padres, es la que utilizó su hija cuando conformó su propia familia y a la que recurre ella luego de su separación. La casilla no sólo se presenta como un recurso familiar que se trasmite en las generaciones de la casa, sino que también porta la memoria familiar, actualiza recuerdos y afectos.

El padre de Rosa trabajaba en la empresa de aeronavegación Austral y “comoganababien, pudo pagar ellote” y“construirla casa”.Según su evocación, y remitiendo al trabajo estable de su padre afirma "antes era más fácil, ahora cuesta un montón, antes se podía construir, ahora no, es una lucha".

Con este ingreso, lograron construir una casa de materiales sólidos que está compuesta por "tres habitaciones y cocina comedor, lavaderoy el baño". Mientras, ella habita la casilla: "Yo atrás duermo con mi hijo". La casilla es su hogar-dormitorio, mientras la cotidianeidad diurna se produce en la amplia casa de sus padres.

En Rosa aparece un relato que evoca tiempos pretéritos mejores y se representa a sí misma y a la actualidad dentro de una trayectoria descendente. Rosa, incluso, cuando estaba en pareja, no pudo acceder a su vivienda propia, fue inquilina y el alquiler no se sostuvo luego de su separación ${ }^{14}$. La imposibilidad de

13 La casilla, en lenguaje nativo, remite a una vivienda de pronta instalación, prefabricada, constituida por materiales de cemento premoldeado o madera. El verbo plantar remite a este momento breve de colocación de la casa.

14 En las referencias a su vivienda alquilada la entrevistada muestra una relación de externalidad. Si bien en la entrevista afirma que fueron elementos económicos los que le impidieron continuar alquilando, pensamos que el desapego afectivo es un componente a tomar en consideración. 
mantener su papel de locataria la hace volver al terreno de la casa de sus padres:

P: Y cuando te fuiste a vivir vos ¿le hicieron alguna ampliación [a la casilla]? R: No, está igual desde que la planté. Porque cada vez que llueve se inunda, entonces para qué voy a adelantar algo que no me sirve. Porque nos llenamos de agua, hasta la mitad de ahí (indica unos $10-15 \mathrm{~cm}$ ). Porque hay muchas napas acá. Vos querés hacer un pozo y se llena de agua.

Resulta llamativo que la entrevistada, exponiendo esta carencia infraestructural de la casa y ante las inundaciones frecuentes de aguas servidas mantenga su rutina nocturna de dormir en esta casilla, cierto resguardo autonómico parece emerger como necesario. Atento a las dificultades mencionadas, el entrevistador le pregunta qué modificación le gustaría realizar en su casa:

$\mathrm{P}: \mathrm{Y}$ de tu casa, ¿qué es lo que cambiarías?

R: No, nada, no, porque se perdería el recuerdo de mi mamá.

P: Claro... ¿ Te gustaría solamente ponerle el techo?

R: Sí, ponerle el techo y construir arriba, porque abajo nos llenamos de agua cada vez que llueve, nos inundamos. Porque hay muchas cloacas.

Ante el asombro del entrevistador, Rosa afirma la relevancia de mantener el aspecto de la casilla para no perder el recuerdo de su madre: la casilla como objeto transicional actualiza el amor materno, se enlaza al primer hogar y lo circunda... la relación entre las personas expresada en las cosas emerge con firmeza. El valor simbólico se manifiesta en su plenitud e, incluso, se presenta en forma tensionada con el valor de uso.

Entendemos que ante las dificultades para acceder a su propia vivienda, Rosa construye un vínculo melancólico con la casilla que habita, cuyo apego enmascara la imposibilidad de independencia y la hace transitar por una solución de compromiso entre la casa paterna actual y sus recuerdos de infancia.

\section{-Viviana: la vivienda, redes y quebranto}

Viviana tiene 50 años, está terminando la escuela secundaria mediante el plan Fines ${ }^{15}$ y es cooperativista del Programa Argentina Trabaja ${ }^{16}$. Vive con tres hijos y su marido (está casada en segundas nupcias) en una casilla que se ubica en la parte trasera del terreno paterno; en el mismo terreno habitan otros dos hermanos con sus familias y se emplaza un local donde su familia desarrolla un pequeño emprendimiento comercial. La vivienda sólida fue adquirida y construida por su padre, un trabajador metalúrgico que incluso adquirió otro terreno y construyó otra vivienda en las cercanías. La llegada al barrio y la construcción de la casa constituye el cierre de un período difícil para la familia, pues la familia paterna venía huyendo de la Segunda Guerra Mundial.

15 Plan de terminalidad educativa para jóvenes y adultos.

16 Programa social de empleo destinado a quienes tienen dificultades para insertarse en el mercado de trabajo. 
Viviana es una de las primeras pobladoras del barrio. Su padre es migrante europeo azotado por la guerra. En su evocación recuerda la transformación de esta zona, en la que se criaba ganado, convirtiéndose hoy en un territorio densamente poblado.

\section{P: ¿Tu papá cómo llegó? \\ R: Mi papá era migrante, soy hija de alemanes, vino hace 50 años. (...) Compró acá, porque mis abuelos emigraron por la Segunda Guerra Mundial. Y... bueno, a raíz de unos conocidos averiguaron sobre estas tierras, compraron acá... empezaron a edificar y así se empezó a poblar este barrio.}

Como en la mayoría de los entrevistados, el ingreso al barrio se produce mediante redes de socialización territorializadas. Se llega a este espacio social mediante algún mediador que ya vive y conoce el lugar. La llegada al barrio posibilita el fin de la huida de la guerra. Este comienzo interpela fuertemente a la familia y dota a la vivienda de una investidura especial.

Con una enérgica remisión al esfuerzo personal y con voluntad de progreso económico, Viviana evoca a su padre como trabajador metalúrgico que logró comprar su terreno y compró otro terreno más, construyó dos viviendas y un local comercial. La familia de origen de Viviana parece condensar la promesa de trayectoria social ascendente propia de mediados del siglo pasado y la búsqueda de la transmisión intergeneracional:

\footnotetext{
P: Bien. Y este... Me dijiste, es de tu papá. ¿Tu papála construyó? ¿La construyó para vos, te la presta, alquilás...?

R: No... A ver, cómo te explico. Mi papá falleció y mi mamá vive en la otra esquina, nosotros todos nacimos y crecimos ahí. Después compró este terreno. Edificó aquí para nosotros. Y bueno... En la parte de atrás vivo yo, en la parte de arriba vive mi hermano, abajo está compartida porque está el local y vive mi otro hermano.

P: O sea que están en familia.

R: Sí, pero cada uno hace la suya. Porque mucho amontonadero, como que también se complica.
}

Sin embargo, las posibilidades de progreso de la entrevistada se vieron limitadas. Las redes territoriales y el andamiaje de sus progenitores serán los sostenes de reemplazo para esta entrevistada que ve coartados sus itinerarios laborales y habitacionales. En este plano esto se expresa en la subdivisión parcelaria y la cohabitación, ambas emergentes en su relato. En el terreno donde vive Viviana habitan trece personas y se lleva adelante un emprendimiento comercial; en su caso si bien el origen de la hechura de la vivienda posee un componente épico, la cotidianeidad de hacinamiento corroe la investidura de la vivienda propiciando una distancia entre sus recuerdos de origen, las expectativas de sus padres y la actualidad.

Esta entrevistada, afirma, sin embargo, que a pesar de que "cada uno hace 
la suya" preferiría vivir sola, pero la falta de recursos económicos y el quiebre de otras alternativas impiden su independencia:

R: Si tuviera la posibilidad me iría. Porque nosotros somos cinco hermanos y está la disputa entre hermanos, ¿viste? Para colmo yo soy la mayor y siempre que aparece un problema la que tiene que ocuparse soy yo.

Este aspecto la induce a buscar alternativas de construir su propio lugar. El deseo de autonomía aparece fuertemente. En este sentido, Viviana afirma que luego de su separación, durante el período que estuvo sin pareja, intentó acceder a un terreno, participando de una ocupación de tierras en un barrio vecino:

R: Yo llegué a tomar una tierra en La Matera.

P: En La Matera. ¿Y cómo fue la experiencia?

R: Muy fea. Porque salí de ahí corriendo. Porque cayó un nene en un brasero. Y yo había ido con mis hijos (...) me asusté mucho al verlo... No podíamos salir ni entrar porque estaba todo custodiado por la policía. Te pegaban, te pegaban muy mal. No respetaban si estabas embarazada, si había chicos, si eras grande, si eras joven. Y era palo que iba, palo que venía... Y bueno, esa vuelta se había quemado ese nene, se cayó en un brasero porque hizo mucho frío... Y bueno, yo me asusté porque mis hijos eran inquietos, y bueno, salí, me vine. Me vine de vuelta a la casa de mi mamá.

Tal como aparece en el relato, la entrevistada entiende que la toma de tierras es una forma legítima de acceder a la vivienda e intenta avanzar en el camino de obtener su terreno mediante la ocupación; sin embargo, la vehemente represión llevada adelante por las fuerzas de seguridad y las condiciones riesgosas de vida durante el proceso de toma produjeron un quiebre en su derrotero. En una disyuntiva trágica, su propio resguardo físico y el de sus niños prevaleció ante el deseo y la necesidad de su propia vivienda. La entrevistada no logra poner en acto el deseo de tener su propia casa, pues las circunstancias sociales intervienen quebrando la acción.

\section{-Alberto: la vivienda y los deseos en tensión}

Alberto tiene 59 años y no finalizó la escolaridad primaria. Trabajó treinta años en la construcción. Actualmente, por un problema de diabetes, perdió parcialmentela visión y se encuentra jubilado. Es oriundo de Tucumán yllegóal conurbano a fines de la década del ' 70 en busca de empleo. Habita una vivienda adquirida informalmente a fines de los ' 80 . Esta se asienta en un terreno que había sido tomado a comienzos delos ' 80 . La vivienda fue "mejorada” mediante el trabajo de su yerno. Actualmente se encuentra terminada, aunque le faltan detalles menores (cerámica en el patio, pintura, etc.). En la casa vive con su mujer, su hija y su nieta. 
En los varones mayores, es la búsqueda de empleo la que los atrae hacia esta zona. Esta llegada se suele producir mediante lazos laborales y/o parentales. Este es el caso de Alberto:

$\mathrm{P}:$ ¿Vino con algún familiar para empezar el trabajo?

R: Yo tenía a mi hermano que vivía acá. Él fue allá a Tucumán, y me dijo “¿Qué andás haciendo vos acá?", "Nada, estoy esperando a ver si...", "Y no, vamos allá, si allá hay trabajo, podés trabajar”, "Y, pero, ¿y adónde voy a vivir?”, "Vas a vivir conmigo, en mi casa”, "Bueno, vamos". Y él me trajo. Después entré en una empresa. Trabajémás de un año ahí en esa empresa, después ya me independicé. Después ya me fui de la casa de mi hermano, me fui solo, y así...

Alberto llega a la casa de su hermano y luego se va a vivir con su mujer, Zulema, que ya tenía una vivienda heredada de su anterior pareja. Años más tarde, mediante una indemnización, recibe un ingreso que le posibilita comprar su vivienda actual:

R: Porque yo había cobrado una plata. Yo estaba enloquecido, quería comprar un auto cero kilómetro. Y mi mujer me dice: "No, vamos a comprar una casa, vamos a comprar un terreno, para la hija, que es la única que no tiene nada". "Bueno, vamos a comprar". Y vine acá, y el señor que vivía acá se estaba por ir al Chaco. Se quería ir al Chaco. "Así que va a vender el terreno, la casa”. "Sî", me dijo, “Te la vendo". "Bueno, te la compro”. Y se la compramos.

El lugar de las mujeres dentro de la pareja en la adquisición de la vivienda propia es un tema que no podemos abordar en este trabajo, pero en el relato queda en evidencia la centralidad que este valor tiene para su esposa, Zulema, y en forma mediada, paraél; mientrasélpretendía comprar un auto, ella solicitaba una vivienda; en su relato el argumento principal es la importancia de que su hija tuviera una vivienda garantizada. La trasmisión intergeneracional del inmueble emerge una vez más, pero brinda una tonalidad diferente en la memoria de Alberto.

La adquisición de la vivienda se produce en efectivo y al contado, dentro de una modalidad informal, como un "arreglo rápido entre vecinos". Ese terreno había sido tomado por la familia vendedora, durante los primeros años de la década del ' $80^{17}$; cuando Albertolo compra, fines delos' 80 , ya estaba en proceso la regularización dominial.

A pesar de que la familia de Alberto compra el terreno con la vivienda en construcción, en la representación del entrevistado, el proceso de elaboración de la casa fue arduo, caro y demorado:

$\mathrm{P}:$ ¿La tuvieron que hacer ustedes?

R: Nosotros hicimos todo. Nosotros pusimos las rejas.

R2 (respuesta de Zulema, la mujer del entrevistado): No había nada acá, era un ranchito, con una puerta de cajón.

17 Este proceso de toma de tierras fue estudiado por Inés Izaguirre y Zulema Aristizabal (1988). 
R:Y ahora, es una casa grande. (...) Lo que nosotros tuvimos que hacer es mejorar. Mejorar ciertas cosas, poner el piso que no había. Terminar el baño. Tuvimos que terminar todos los pisos, remodelar la cocina. Y alargar un poco el techo. Lleva un tiempo. Así que... Lo vamos haciendo de a poco. La casa se compone acá de un comedor de cuatro por cuatro, tiene dos dormitorios de tres por tres. Un baño de dos por uno, y un antebaño. Y una cocina de cuatro por cuatro.

P: Muy grande, muy confortable. ¿Y ustedes mismos hacían los arreglos o...? R2: No, mi yerno los hacía.

R: Mi yerno, el marido de mi hija más grande, se da maña para todo. ¡Todo! Nosotros gracias a Dios tenemos todo. Tenemos baño instalado, tenemos la cocina instalada. Todo, nos hizo el yerno. Instalamos el baño, instalamos la cocina.

Alberto menciona con detalle cada una de las habitaciones que conforman su casa. Precisa su tamaño, se enorgullece de su amplitud. Afirma que "les llevó muchos años" pero finalmente lograron construir la vivienda esperada. En la vivienda habitan tres generaciones: la cohabitación familia o la variedad familiar se encuentran fuertemente presentes en los sectores populares ${ }^{\mathbf{1 8}}$; no obstante, en este caso no se vislumbra hacinamiento ${ }^{19}$.

Es llamativo, en relación a la producción de la vivienda, sin embargo, que sea el yerno el que construye la casa, pues el entrevistado también tiene el oficio de la construcción pero sólo aparece como el promotor, el financista y el coordinador de la hechura. La producción de la vivienda se transluce como un intercambio de reciprocidades:

R: La otra vez se me rompió esa ventana, le digo: ¿Podés venir a ponermela cortina? “¿Cuánto le puedo cobrar?”, me dice, me jode. “¿Qué va a venir a cobrar?” le digo. ¿Cuánto vale mi hija?

La hija, un poco en broma, un poco en serio, asoma como la garante de la intermediación. La colaboración familiar tiene ahí un sustento y la remisión a la asistencia por mediación de dones no resulta forzada.

Ahora bien, entre la pareja, la continuidad de los detalles faltantes en la vivienda es motivo de debate. Zulema, que irrumpe intermitentemente en la situación dela entrevista, alude a alguna carencia en relación a la casa, mientras nuestro entrevistado se refiere al deseo de emprender otro tipo de acciones:

P: ¿Y hay algo que le cambiarían?

R: No, no...

R2: ¡No quiere cambiar más nada!

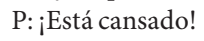

R: Sí, sí, lo que me gustaría sería terminar... Poner una cerámica acá en el piso [señala el piso del patio de la casa que es de cemento], todo esto, que quedaría un poco mejor... Lo que pasa es que a veces vos decís... "Bueno, me voy a

18 La pregunta en torno a si los hogares compuestos por más de dos generaciones suponen una cohabitación de familias o un arreglo familiar diferente excede este trabajo, pero resulta pertinente tomarlo en consideración.

19 En una casa de 90 metros cuadrados, compuesta por cuatro ambientes, pueden habitar cuatro personas de manera confortable. 
ocupar de mí, me voy a ocupar de mi mujer, nos vamos a ir a cenar, vamos a ir a salir...” Nosotros nunca salíamos a cenar. Ella quiere todo en la casa. “¡Dejate de joder! Vamos a cenar, vamos al cine... Vamos... Vamos aunque sea por ahí a caminar, a comer un helado.... Cosa que no hicimos antes. Antes no lo hicimos porque estábamos avocados a esto. A la casa, que había que trabajar, que veníamos tarde, que teníamos sueño, que estábamos muy cansados... Y ahora que podemos, le digo: "Bueno (...), por lo menos vamos a comer un helado, vamos a comer unas pizzas, vamos a caminar”.

Es interesante atender al desplazamiento y la inversión que produce el entrevistado. Alberto se está quedando ciego, no puede trabajar y siente que dedicó mucha de su energía, de su tiempoy de su dinero en la casa, está orgulloso con el resultado, pero evalúa que se podría dar por cerrada la etapa. El entrevistado espera tener tiempo y dedicar dinero para "darse un gustito", para salir aunque sea para realizar a actividades poco onerosas. El disfrute, las salidas ylos paseos resultan una acción extracotidiana para este entrevistado, quien afirma haber pasado toda su vida avocado a la construcción de la casa y ahora - jubilado y enfermo- espera poder hacerlo. La tensión afectiva que produce el esfuerzo de construcción de la vivienda se expresa patentemente en este entrevistado.

\section{- Fermín, la vivienda y el disfrute de la vida}

Fermín tiene 60 años y es chaqueño, llegó a la zona por motivos laborales. En relación a sus estudios, sólo completó la escolaridad primaria. Fermín tuvo una trayectoria laboral heterogénea y sinuosa, pero con la colaboración económica de su mujer - que también trabaja - y actualmente de su hija, lograron construir una amplia vivienda a la que le faltan terminaciones. La casa fue financiada por él y su mujer; el terreno fue adquirido a finales de los ochenta mediante una operación informal mediada por redes de sociabilidad. Vive con su mujer y su hija, ya adulta.

Fermín llega al barrio por un compañero de trabajo y es a partir del vínculo con él que logra que le vendan un terreno con una casilla a pagar, una parte al contado y en efectivo y otra parte en cuotas.

P: Y cuando llegó acá: ¿Cómo accede a la casa? ¿Empezó a alquilar? R: Yo sabía que se podía, mi señora trabaja, y sabía que se podía más o menos pagar un alquiler y seguir ahorrando, y tuve suerte porque yo iba juntando unos pesos, ahorrando, y conmigo laburaba un muchacho, que era el padre de él; él vino muy chiquito del Chaco, y cuando le pregunto el apellido, y el padre era un vecino mío, él era chiquito y no sabía. Cuando yo hablé, le pregunto allá por tu barrio no hay nada para alquilar, y él dice voy a empezar a mirar. Y ahí justo donde yo vivo, vivía una prima lejana de él, entonces qué pasa, yo no tenía todo el dinero, y no era fácil vender todo al contado entonces la señora era viuda, y como yo era amigo del consuegro de él, fui pagando de a poco, no era fácil. Para colmo, época de Alfonsín. Aparte yo hice un trato con ella para darle la plata, y en un momento yo no llego y le digo véndalo, pero ella me esperó. 
Una vez más emerge la matriz relacional como garante de los intercambios. No sólo es por el conocimiento intermediado que Fermín consigue comprar su terreno en cuotas, sino que consigue que lo esperen en una situación de dificultad para cancelar la mensualidad. El lazo laboral/territorializado aparece como recurso asegurador.

La hechura de la vivienda comienza, una vez más, por la casilla; este es el primer eslabón en la biografía de esta morada y la que posibilita la sutura de la necesidad agilizando el usufructo.

P: ¿Tuvo que arrancar la casa desde cero?

R: No, yo empecé viviendo en la casilla... y después fuimos haciendo la casa. $\mathrm{P}:$ ¿Es de material la casa?

R: Sí, de material. (...) Todavía conservo mi casilla, la casilla es nuestra vida, le digo a mi hija, esta casilla la voy guardar para tenerla de recuerdo. (Risas.)

No obstante, una vez más, la casilla aparece con un excedente que sobrepasa aquel primer lugar ya en desuso; este emerge como un recurso para las nuevas generaciones, como un soporte de la memoria y un monumento. Afectivamente investido, este recurso actualiza la historia y preserva el trofeo. Cabe detenerse en este objeto que se produce por la inminente necesidad, que desde su comienzo se espera sea transitorio, pero no sólo constituye un momento en la historia social de la vivienda propia ${ }^{20}$, sino que permanece con múltiples sentidos.

La producción de la casa de material, como en los demás entrevistados, se fue realizando de a poco y mediante el esfuerzo mensual provisto a partir del salario de la pareja. Como en el caso anterior aún hoy la vivienda está parcialmente completa, sin embargo el proyecto de Fermín es bastante ambicioso:

$\mathrm{P}: \mathrm{Y}$ ¿cómo es su casa actualmente? ¿Cuántos ambientes tiene?

$\mathrm{R}$ : Y todavía me falta terminar, arriba está prácticamente terminado y abajo falta terminar.

P: ¿Y cuántos ambientes tiene?

R: Hay dos dormitorios, dos baños, un living, un comedor, la cocina y la terraza. Abajo falta terminar el segundo baño, el baño de las visitas. Lo restante de la vivienda está finalizado aunque faltan detalles.

La casa construida excede la amplitud media de la vivienda de los sectores populares. Hay un plus que si bien no selogra completar, parece vincular el proyecto de vida con el proyecto de casa. En un momento de la entrevista Fermín dice: "la casilla es nuestra vida", remitiendo a la memoria familiar bajo el soporte de esta vivienda inaugural, posteriormente el entrevistado afirma "mi casa, es todo" identificando la totalidad de su ser individual y familiar a la referencia

20 Acerca de la biografía y las transformaciones de los valores de los objetos, véase: Appadurai (1991). 
material de la vivienda ${ }^{21}$. Esta forma de alocución nos permite inferir que la incompletud de la casa, es también la energía vital, el motor del deseo que hace que Fermín siga trabajando, siga viviendo, siga deseando.

En Fermín el placer en torno al uso actual de la vivienda propia aparece de forma particular:

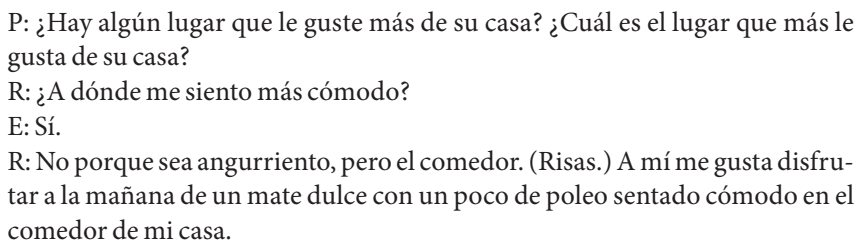

El comedor, el mate, la comodidad, la vivienda construyen una red de disfrute que aparece anudada en las representaciones del entrevistado. El mate, para los rioplatenses no es sólo una infusión, es un ritual que porta una serie de símbolos ${ }^{22}$. La asociación entre estos componentes del placer contiene una fuerte carga afectiva que se produce en la vivienda. El disfrute, para este entrevistado es cotidiano y doméstico, y se inscribe en el seno de la propia morada que orgullosamente manifiesta haber logrado construir.

\section{REFLEXIONES FINALES}

La estrecha responsabilización estatal en torno al derecho a la vivienda impone una serie de desafíos a todos los habitantes. Estos conllevan extraordinarios esfuerzos para los sectores más empobrecidos de las clases trabajadores, cuya puesta en acto se realiza por la investidura que este bien contiene. Incluso, en las experiencias no exitosas se identifican esfuerzos tendientes a la producción de la morada. Este laborioso proceso posee un fuerte anclaje en redes territorializadas de cooperación que brindan un recurso central para la dimensión habitacional y la vida cotidiana.

Los casos analizados en este artículo muestran que dentro de un mismo territorio barrial, entre sujetos de vecindad, se puede encontrar itinerarios diferenciales que se ligan a aspectos de género y etarios. No obstante, entre itinerarios semejantes en relación a las dimensiones mencionadas, también aparecen formas diferentes de representar sus logros y sus penurias habitacionales.

Intrínsecamente ligado a su generación y a su condición de género, Rosa y Viviana no lograron construir una vivienda propia separada del terreno de sus padres. Ambas habitan una casilla en el espacio posterior de la vivienda paterna. Noobstante, susitinerariosylasformas representacionales que expresan poseen

21 El trabajo de García García explora esta analogía entre el ser y la vivienda bajo la noción de identidad (2005).

22 En torno a esta cuestión, se puede ver el libro de Barretto (1989). 
diferencias relevantes. Rosa alquila durante un breve lapso pero no logra mantener ese inquilinato; regresa a la casilla emplazada en el terreno paterno y debe soportar las inundaciones y el deterioro de la vivienda fabricada con materiales de corta duración. Sin embargo, su apego a ese bien le impediría modificarlo: el recuerdo de su madre fallecida se expresa en la materialidad de esa vivienda. La melancolía contiene su vínculo con esta casa. Los padres de Viviana llegan al barrio huyendo de la guerra, ellos lograron construir dos viviendas cercanas, sin embargo Viviana no logró construir una casa propia; habita la casilla trasera emplazada en el terreno paterno; en ese terreno habitan muchas personas y esta actualidad hacinada hizo que Viviana avizorara un camino para intentar tener su propio lugar de vida. Mediante su participación en una toma de tierras trata de buscar un carril alternativo a su situación de carencia, pero la represión y los peligros suscitados en la toma la hacen desistir. Viviana vuelve a su casa paterna, pero trae consigo un quiebre en su proyecto y sus expectativas.

El quebranto y la melancolía son dos figuras que condensan experiencias que aparecen en quienes no lograron acceder viviendas propias y autónomas. Los casos estudiados nos permiten construir dos núcleos figurativos de una tipología en proceso. Ambos se ligan a la imposibilidad de acceso a una morada independiente de su familia de origen, sin embargo las articulaciones entre los componentes de las representaciones sociales emergentes difieren en forma significativa. En ambos la forma en que describen la materialidad de las viviendas que habitan aparece como deteriorada; el amparo y la protección habitacional que las contiene se ve avasallada, vulnerando el valor de uso de la vivienda, es por ello que buscan alternativas para salir de esa morada; no obstante, los intentos se ven quebrados por circunstancias sociales, ante esta situación se ven compelidos a retornar, dicho retorno se significa de formas diferentes. Uno de los núcleos figurativos que hemos construido a partir del caso de Viviana cierra un círculo de quebranto: el déficit de valor de uso no se resignifica como valor simbólico de remplazo, este hogar va perdiendo su investidura épica; en contrapunto, el otro núcleo figurativo, ejemplificado a partir del caso de Rosa, por el contrario, resignifica el valor simbólico de la casilla de origen bajo la evocación melancólica como objeto transicional. Como hipótesis para seguir indagando se puede pensar que la representación social del quebranto se liga a una sobrevaloración de la propia vida por sobre el valor de la vivienda; mientras que el retorno melancólico se asienta en una revaloración de la vivienda, incluso en ruinas, como portador de la memoria familiar.

Alberto y Fermín hoy tienen cada uno de ellos sus propias viviendas. Ambas tienen pequeños faltantes, pero ninguno de ellos resulta perjudicial parala vida cotidiana. Ambos compraron informalmente, a partir de lazos territorializados, los terrenos/casas; los dos proyectaron viviendas amplias y confortables y, con un largo proceso de trabajo, hoy se enorgullecen de lo conseguido. Sin embargo las formas en que emergen las representaciones sobre este aspecto 
poseen diferencias de relevancia. Alberto hoy está jubilado y enfermo, en su relato afirma que pasó su vida dedicándose a la construcción de la vivienda; sus dichos reflejan cansancio y agobio. En Alberto la carga afectiva que proporcionaba este plus energético destinado a la vivienda parece haberse agotado; el entrevistado quiere vivir otras experiencias placenteras. En él aparece la necesidad de pasear, el derecho al ocio extradoméstico emerge como un deseo que quiere brindar(se) durante el último tramo de su vida. Por el contrario, en Fermín el trabajo de la vivienda y su demorado y agotador proceso se revalorizan intrínsecamente; este entrevistado construye una fuerte analogía entre su ser y su vivienda; su orgullo y su disfrute se encuentran en el logro obtenido y en la cotidiana posibilidad de usufructuarlo.

La tensión y el disfrute son dos figuras que resumen las experiencias que aparecen en quienes lograron construir viviendas propias y autónomas. A partir de los casos de Alberto y de Fermín estamos construyendo dos núcleos figurativos que podrían constituirse en herramientas para nuevas pesquisas. Ambos tienen en común la realización del laborioso y costoso itinerario para el acceso a una morada independiente respecto dela familia de origen. Asimismo, la forma en que se describe la materialidad de las viviendas que habitan aparece como confortable y amplia; el amparo y la protección habitacional que las contiene articula orgullosamente el valor de uso y el valor simbólico de la vivienda, son ambos valores los que se conjugan invistiendo el sentido de la morada en estos dos tipos de representaciones sociales. No obstante, las articulaciones entrelos componentes delas representaciones sociales que constituyen estos tipos difieren de manera relevante. Para uno de ellos, ejemplificado a partir del caso de Alberto, resuelta la utilidad dela vivienda - y atento a otros núcleos de placer ante su propia vejez - la producción de los últimos detalles de la casa pasa a tener una importancia secundaria, expresando una tensión entre el proyecto doméstico y extradoméstico. En contrapunto, el otro eslabón de la tipología, analizada a partir del caso de Fermín, la representación social de la vivienda emerge como el núcleo monopólico del disfrute; ésta, en sus diversas versiones, aparece como la materialización del propio proyecto de vida. Cabe decir que, entonces, el valor de la propia vida y el valor de la vivienda propia se enlazan en forma inversa en cada uno de estos tipos de representaciones sociales: mientras en uno aparecen ligadas, en el otro emergen como una relación inversa.

Los casos analizados nos brindan algunos elementos para pensar las diversas formas en que aparece investida la vivienda propia. La melancolía, el quebranto, la tensión y el disfrute fueron las figuras estudiadas ${ }^{23}$. Nuevas aproximaciones nos permitirán poner a prueba estos núcleos figurativos que surgieron de este primer trabajo.

23 Durante los días que escribíamos este artículo, fuimos a ver el extraordinario film Aquarius (2016). Esta película brasileña, de Kleber Mendonça Filho, brinda una gran cantidad de sugerencias para pensar la cuestión del apego en la vivienda; no sólo en los sectores más empobrecidos de las clases trabajadores, sino en fracciones sociales medias-altas. 


\section{REFERENCIAS}

Appadurai, A. (1986, 1991). Introducción. Las mercancías y la política de valor. En La vida social de las cosas. Perspectiva cultural de las mercancías (pp. 17-97). México DF: Grijalbo.

Barretto, M. (2006). El mate. Su historia y su cultura. Buenos Aires: Ediciones del Sol.

Bertotti, M. C. (Septiembre, 2015). Entre los grandes relatos y las memorias locales acerca de la última dictadura cívico-militar. Una aproximación a los vecinos de Malagueño de la Provincia de Córdoba. En Seminario inernacional de Políticas de la Memoria. Centro Cultural Haroldo Conti, Buenos Aires, Argentina.

Cravino, M. C. \& Varela, O. D. (2009). Mil nombres para mil barrios. Los asentamientos y villas como categorías de análisis y de intervención. En Cravino, M. C. (org.).Los mil barrios (in)formales (pp. 45-64). Los Polvorines: UNGS.

Di Virgilio, M. (2015). Urbanizaciones de Origen Informal en Buenos Aires. Lógicas de producción de suelo urbano. En Estudios Demográficos y Urbanos, 30, (3), pp. 651-690.

García García, A. (2005). Vivienda, familia, identidad. La casa como prolongación de las relaciones humanas. En Trayectorias, 7, (17), pp. 43-56.

García Canclini, N. (1993). El consumo cultural y su estudio en México. Una propuesta teórica. En García Canclini, N. (org). El consumo cultural en México (pp. 15-42 ). México DF: Conaculta.

Harvey, D. (2014). Contradicción 1. Valor de uso y valor de cambio. En Harvey, D. Diecisiete contradicciones y el fin del capitalismo (pp. 31-39). Quito: Prácticas Constituyentes.

Herzer, H., Di Virgilio, M., Rodríguez, M. C. \& Redondo, A. (2008). ¿Informalidad o Informalidades? Hábitat Popular e Informalidades Urbanas en Areas Urbanas Consolidadas (Ciudad de Buenos Aires). En Pampa, 4, pp. 85-112.

Izaguirre, I. \& Aristizabal, Z. (1988). Las tomas de tierras en la zona Sur del GBA. Buenos Aires: Centro Editor de América Latina.

Jodelet, D. (1986). La representación social: fenómenos, conceptos y teoría. En Moscovisci, S. (comp.) Psicología social II (pp. 469-494). Barcelona: Paidos.

Lara, A. \& Enciso Domínguez, G. (2013). El giro afectivo. Athenea Digital, 13(3), pp. 101-119.

Marx, K. (1867, 2010). La Mercancía. En El Capital, Tomo I (pp. 43-102). EspañaMéxico-Argentina: Siglo XXI Editores. 
Municipio de Quilmes (2011). Censo Social. Resultados definitivos-2010. Quilmes: Fundación Fudeni.

Núñez,P.(2004). Prácticas políticas en un barrio del Gran Buenos Aires Un acercamiento a los criterios de justicia en jóvenes de sectores populares. En Revista Kairos. Revista de Temas Sociales, 8, (4), pp. 1-18.

Murard, N. \& Laé, J. F. (2013). El mendigo, el bandido y el buen trabajador. Ascetismo y hedonismo en las clases populares. En: Castel, R., Kessler, G., Merklen, D. \& Murard, N. Individuación, precariedad, inseguridad ¿Desinstitucionalización del presente? (pp. 87-108). Buenos Aires: Paidós.

Scott, J. W. (2001). Experiencia. En La Ventana, 13, (2), pp. 42-74.

\section{IDENTIFICACIÓN DE LA AUTORA}

María Maneiro es Doctora en Ciencias Humanas, mención en Sociología, por el Instituto Universitário de Pesquisas do Rio de Janeiro (IUPERJ), Brasil. Es Investigadora del Consejo Nacional de Investigaciones Científicas y Técnicas (CONICET), Argentina. Se especializó en el estudio del conflicto social, las espacialidades periféricas y las memorias sociales. Es Profesora de la Carrera de Sociología, Facultad de Ciencias Sociales, Universidad de Buenos Aires (UBA). Dicta cursos de posgrado en distintas universidades nacionales. Publicó los siguientes libros: Como el Árbol Talado (2005) y América Latina Hoje (2008), además de diversos artículos en revistas académicas internacionales.

\section{REGISTRO BIBLIOGRAFICO}

Maneiro, María (2017). Melancolía, quebranto, tensión y disfrute. Representaciones sociales respecto de las experiencias de construcción de la vivienda en los barrios populares periféricos. InMediaciones de la Comunicación, 12(1), 241-259. 